\title{
fMRI in patients with lumbar disc disease: a paradigm to study patients over time
}

This article was published in the following Dove Press journal:

Journal of Pain Research

8 December 201I

Number of times this article has been viewed

\section{Harish A Sharma' \\ Rajarsi Gupta ${ }^{2}$ \\ William Olivero 3}

'Robarts Imaging, University of Western Ontario, London, ON, Canada; ' ${ }^{2}$ niversity of Illinois, Urbana, IL, USA; ${ }^{3}$ Neurological Surgery, University of Illinois UC/Carle Foundation Hospital, Urbana, IL, USA
Correspondence: Harish A Sharma Research Scientist, Robarts Imaging Laboratories, University of Western Ontario, 100 Perth Drive, PO Box 5015 , London, ON N6A 5K8, Canada

$\mathrm{Tel}+\mathrm{I} 5196977933$

$\mathrm{Fax}+15199315260$

Email hsharma4@uwo.ca
Abstract: Low back pain is a common human ailment. It is estimated that over $70 \%$ of the population will experience low back pain that will require medication and/or medical attention. There are many causes for low back pain, one being herniation of the discs of the lumbar spine. Treatment options are very limited. Why patients develop chronic pain especially when there is no known organic cause or when the offending painful stimulus has been removed remains poorly understood. Functional magnetic resonance imaging (fMRI) is a technique that allows researchers to image which regions of the brain that are activated during motor, cognitive, and sensory experiences. Using fMRI to study pain has revealed new information about how the brain responds to painful stimuli and what regions of the brain are activated during pain. However, many of the paradigms used do not replicate the subject's pain or use painful stimuli in volunteers without pain. Also, following patients from their acute phase of pain to the chronic phase with serial fMRI has not been performed. In this study we developed a paradigm that would allow studying patients with low back pain and leg pain including lumbar radiculopathy to better mimic a clinical pain syndrome and to have a method of following patients with this type of pain over time.

Keywords: functional magnetic resonance imaging, low back pain, pain syndrome, chronic pain

\section{Introduction}

Chronic pain affects millions of patients and the treatment options that are currently available are either limited, ineffective, or result in significant side effects. ${ }^{1-3}$ Furthermore, why and how patients develop chronic pain, especially when there is no known organic cause or when the offending painful stimulus has been removed, remains to be fully elucidated. For most pain-associated problems, it is difficult to predict which patients will develop chronic pain. For example, in one study, over $90 \%$ of patients with herniated lumbar discs responded to therapy and their acute pain resolved. ${ }^{4}$ However, in patients with herniated lumbar discs who have a workman's compensation injury claim, as many as $30 \%-60 \%$ go on to develop chronic pain. ${ }^{1}$

Functional magnetic resonance imaging (fMRI) is an imaging technique that allows researchers to investigate and identify the regions of the brain that are activated during motor, cognitive, and sensory experiences. Using fMRI to study pain has revealed new information about how the brain responds to painful stimuli and what regions of the brain are activated during pain., ${ }^{2,3}$ Unfortunately, many of the paradigms that are used in fMRI studies either fail to replicate the subject's pain or painful stimuli is used in volunteers without pain. ${ }^{5,6}$ Moreover, longitudinal fMRI studies that follow patients who develop chronic pain from the acute phase of pain have not been performed. ${ }^{2}$ 
We developed an fMRI paradigm that reliably mimics a clinical pain syndrome in patients who have low back pain and leg pain from acute lumbar radiculopathy and lumbar degenerative disc disease. We believe this paradigm will allow longitudinal fMRI to be used on patients as they progress from acute to chronic pain.

\section{Methods}

Volunteers without a clinical history of pain and no symptoms of back pain were used as controls. Patients who suffered from either acute lumbar radiculopathy or chronic low back pain from lumbar degenerative disc disease were recruited from a local spine center (Carle Spine Institute) and Institutional Review Board approval was obtained from both the hospital (Carle Foundation Hospital) and the university (University of Illinois at Urbana-Champaign) Institutional Review Board committees. All of the patients in the study also had a recent MRI of the lumbar spine prior to the fMRI studies.

The duration, distribution, and level of the patients' pain and disability during resting baseline were rated and recorded by using a visual analog scale (VAS) and the Oswestry disability index. Using the VAS, the patient rated pain from zero to 10 , where zero indicates no pain and 10 indicates unbearable pain. The Oswestry disability index ${ }^{4}$ is a scale that is commonly used to assess how a patient's back pain affects their quality of life via questionnaire with a scale that ranges from zero to 50 , where $0-10$ indicates minimal disability and 40-50 indicates being bed-bound.

Patients were paid remuneration to participate in the study. Even if they could not complete the study, they were compensated for their time. CAD $\$ 50$ to participate in the study and CAD\$25 if they could not complete the study. Patients were instructed to withhold their pain medications for four hours before the fMRI studies. Prior to the actual experiment, the fMRI study session involved the patient filling out an Oswestry questionnaire, which was then followed by placing the patients in a mock MRI with instructions and information about the various maneuvers that would be performed during the fMRI measurement that could elicit pain. All of the subjects were told that they would have to rate their pain immediately before and after each set of maneuvers. Every patient and control subject was told that they could immediately terminate the study at any time.

Pain augmentation maneuvers included breath-holding (Valsalva maneuver), straight leg raising (Lasègue's test) as seen in Figure 1, dorsiflexion of the foot, and tensing the muscles in the painful leg. ${ }^{7}$ After each maneuver, the patients were asked to rate their pain using the VAS during both

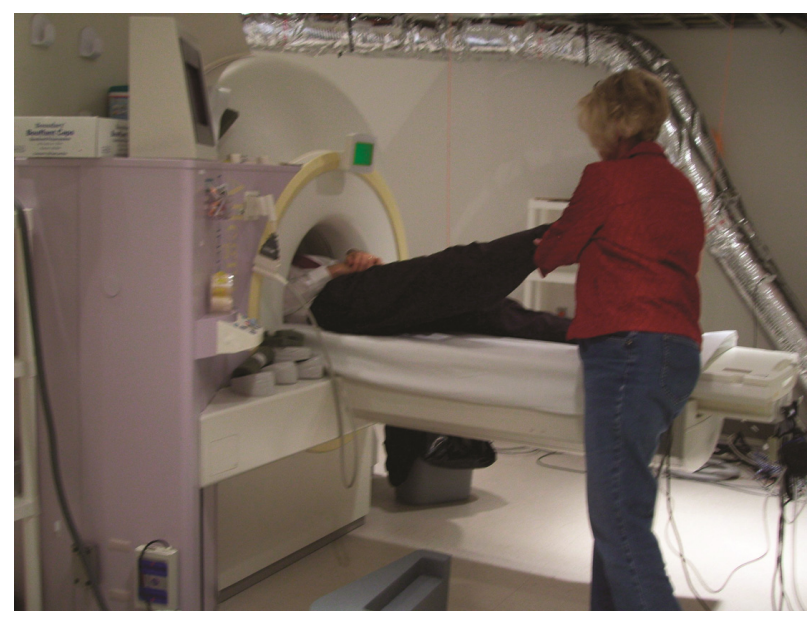

Figure I Straight leg raise maneuver during fMRI measurement.

the resting baseline and immediately after each maneuver was performed. Control subjects performed the same set of maneuvers as the patients who all had pain. All leg maneuvers were performed with the right leg, since this was the most painful extremity in all subjects.

\section{Image acquisition}

Patients were imaged in an Allegra 3T scanner (Siemens Medical Systems, Erlangen, Germany) at the Biomedical Imaging Center at the University of Illinois at UrbanaChampaign using a single-channel quadrature coil. Block design was used for fMRI stimulus design, which consisted of 20 seconds of the pain-eliciting maneuver and 20 seconds resting baseline, repeated ten times. A T2*-weighted EPI sequence $\left(\mathrm{TR} / \mathrm{TE} / \mathrm{FA}=2000 \mathrm{~ms} / 30 \mathrm{~ms} / 77^{\circ}\right)$ was used to acquire whole brain volumes (32 slices) at a voxel dimension of $3.8 \times 3.8 \times 4 \mathrm{~mm}^{3}$. High-resolution T1-weighted anatomical images were acquired with an MP-RAGE sequence.

\section{Image processing and statistical analysis}

fMRI data were analyzed using BrainVoyager QX (Brain Innovation, Maastricht Netherlands). ${ }^{8}$ The standard sequence of preprocessing steps performed to analyze the fMRI data includes slice-scan time correction, high-pass filtering, and spatial smoothing. Three-dimensional (3D) head motion correction was performed to detect and correct for small head movements and estimated translation and rotation parameters were also calculated and inspected. The anatomical data was corrected for spatial intensity inhomogenities and then the data was resampled to $1 \mathrm{~mm}$ resolution and transformed into anterior posterior commissure and Talairach standard space. ${ }^{9}$ The fMRI data was coregistered with the subject's $3 \mathrm{D}$ 
anatomical data and normalized in order to perform the analysis in Talairach space.

For each subject block of data, a Brain Voyager protocol file was derived representing the onset and duration of the tasks for the different conditions and maneuvers. In order to account for hemodynamic delay and dispersion, each of the predictors was derived by convolution of an appropriate boxcar waveform with a double-gamma hemodynamic response function. Using hypothesis-driven voxel-wise standard analyses (generalized linear model), we tested for overall task-related effects during the fMRI measurements.

\section{Results}

Nine patients were recruited, seven of whom completed the study. The two patients who could not complete the study were too large for the Siemens MRI and were excluded from the study during the mock MRI phase. One patient's data was excluded due to motion degradation. Three patients had acute radiculopathies from herniated discs with pain for less than 3 months duration. Three healthy female volunteers were used as controls. The average age of the subjects was 35 years. Table 1 gives the patient demographics, duration of pain, lumbar MRI findings, baseline pain ratings, pain during the straight leg raise (SLR) maneuver, and Oswestry score.

All the subjects tolerated the sessions without any difficulty and all the phases of the fMRI paradigm were completed. The SLR maneuver caused a two-point or greater change on the VAS during the fMRI sessions and was the most likely maneuver to activate regions of the brain in the pain matrix. We report the group analysis results $(P=0.05$, corrected) as figures that show the activated regions in the brain as a result of low back and leg pain. Figure $2 \mathrm{~A}-\mathrm{D}$ represents the summed data for the pain subjects during the SLR maneuver. The images show activation in the anterior cingulate gyrus, bilateral insular regions, right thalamus and basal ganglia, sensorimotor regions (greater on the left side), and anterior cerebellar vermis. Figure $3 \mathrm{~A}-\mathrm{C}$ shows that the activation regions in the controls during SLR are the sensorimotor region (representing the leg and back) and the cerebellar vermis. Table 2 shows the Talairach coordinates for the activated brain areas.

\section{Discussion}

Low back pain is a common human ailment and it is estimated that over $70 \%$ of the population will experience lumbar pain that will require medical attention. ${ }^{10}$ Pain is a unique and complex experience that is processed in the brain through sensory, emotional, and cognitive circuitry. Although the mechanisms of how the brain processes and experiences pain are being actively investigated with fMRI and positron emission tomography studies, it is still unclear as to why certain types of painful experiences activate specific regions of the brain and not others. ${ }^{3}$ Numerous fMRI studies involving pain have demonstrated activation of specific regions of the brain that have come to be referred to as the pain matrix. ${ }^{2,3}$ The pain matrix includes the primary sensory cortex (S1), secondary sensory cortex (S2), bilateral thalami, cingulate cortex, frontal lobes, amygdala, and insular regions. Not all pain studies activate all of these regions. The regions of the brain that were activated in the patients with low back and leg pain in our study were typical regions in the pain matrix. These included S1, cingulated gyrus, amygdala, thalamus, and insula. S1 was also activated in the controls but to a lesser degree.

The main purpose of our study was to determine which maneuvers that augment back and leg pain could be performed during fMRI without causing problems related to motion artifacts and without causing undo discomfort. fMRI studies require a fluctuation of the pain level. Constant pain cannot be studied using fMRI even if it is severe.

The SLR maneuver was the most reliable maneuver that activated regions of the pain matrix. This maneuver consistently caused a 2-4 point increase in the VAS. Although there were some problems with motion, these artifacts could be readily

Table I Patient demographics, MRI findings, type and duration of pain

\begin{tabular}{|c|c|c|c|c|c|c|}
\hline & $\begin{array}{l}\text { Gender, age, } \\
\text { handedness }\end{array}$ & $\begin{array}{l}\text { Lumbar MRI } \\
\text { findings }\end{array}$ & $\begin{array}{l}\text { Duration of pain } \\
\text { in patients }\end{array}$ & $\begin{array}{l}\text { Pain at rest VAS } \\
\text { scale }\end{array}$ & $\begin{array}{l}\text { Pain during SLR } \\
\text { VAS scale }\end{array}$ & $\begin{array}{l}\text { Oswestry low } \\
\text { back pain scale }\end{array}$ \\
\hline Subject I & $F, 44, R$ & DDD & Chronic & I & 4 & 17 \\
\hline Subject 2 & $\mathrm{~F}, 4 \mathrm{I}, \mathrm{R}$ & DDD & Chronic & 7 & 10 & 35 \\
\hline Subject 3 & $F, 54, R$ & DDD & Chronic & 7 & 9 & 36 \\
\hline Subject 4 & $\mathrm{~F}, 5 \mathrm{I}, \mathrm{R}$ & HNP & 2 months & 5 & 8 & 34 \\
\hline Subject 5 & $\mathrm{~F}, 29, \mathrm{R}$ & HNP & 2 months & 7 & 10 & 30 \\
\hline Subject 6 & $M, 54, R$ & HNP & I month & 1 & 4 & 30 \\
\hline
\end{tabular}

Abbreviations: DDD, degenerative disc disease of the lumbar spine; HNP, herniated disc at L4-5 or L5-SI; MRI, magnetic resonance imaging; VAS, visual analog scale. 

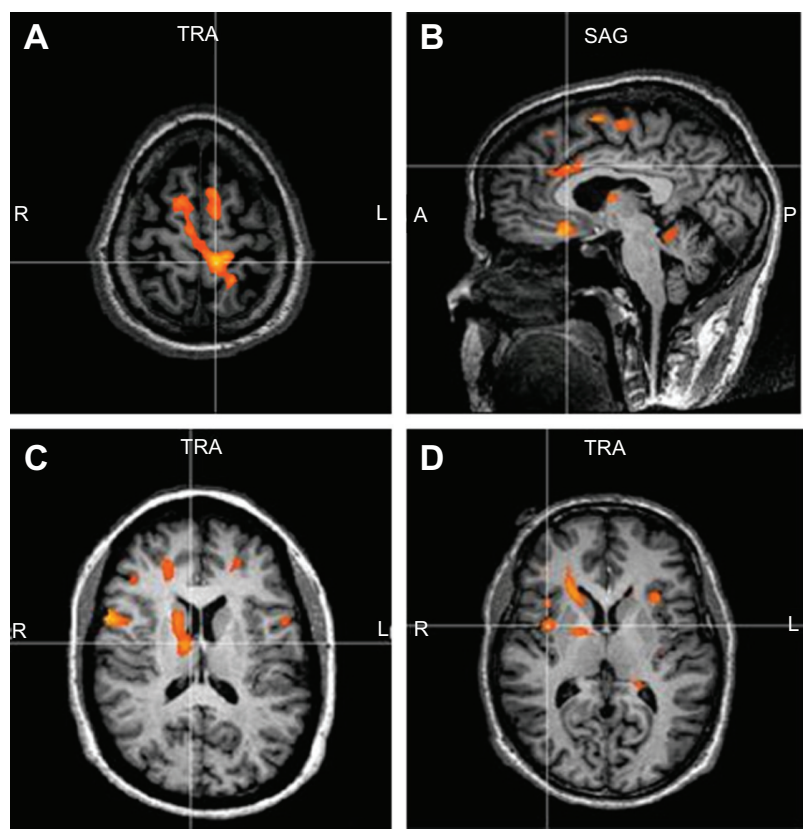

Figure 2 Demonstrates the activated regions of the patients during the SLR maneuver. There is activation in the leg sensory motor region SI (A), anterior cingulate gyrus (B), thalamus and caudate (C), and insular regions (D). Abbreviation: SLR, straight leg raise.

addressed and quantitatively corrected. Dorsiflexion of the foot and tensing the leg muscles less reliably caused a change in the VAS and less reliably activated the pain matrix. The Valsalva maneuver caused diffuse activation of the brain and therefore could not be used to study low back pain with fMRI.
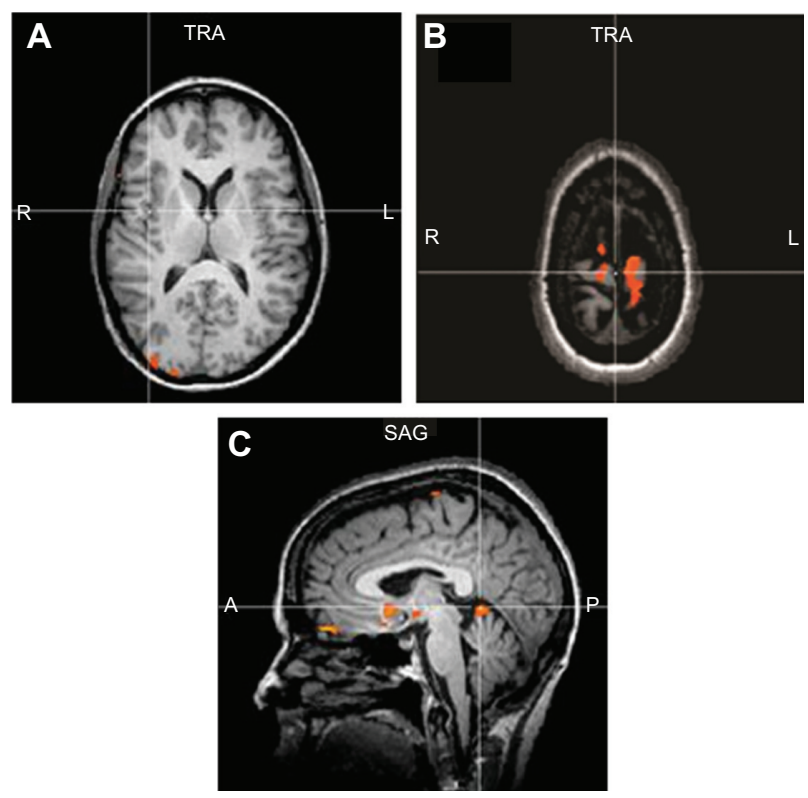

Figure 3 Demonstrates no activity in the pain regions $(\mathbf{A})$ of the controls during the SLR maneuver. There is activation in the leg sensory motor region SI (B) and anterior cerebellum $(\mathbf{C})$.

Abbreviation: SLR, straight leg raise.
Table 2 Talairach coordinates for the activated brain areas

\begin{tabular}{llll}
\hline Activated brain areas & $\mathbf{X}$ & $\mathbf{Y}$ & $\mathbf{Z}$ \\
\hline Motor area & -10 & -32 & 59 \\
Anterior cingulate & 5 & 24 & 27 \\
Thalamus & $\mathrm{II}$ & -10 & 14 \\
Caudate & 13 & 11 & 14 \\
Insula & 38 & 2 & 8 \\
\hline
\end{tabular}

There have been several fMRI studies involving patients with low back pain. Moseley and colleagues ${ }^{11}$ demonstrated decreased fMRI activation in a single patient with chronic low back pain during performance of an abdominal task after pain physiology training. He hypothesized that the decrease in activation which occurred in regions of the pain matrix may reflect a reduced threat value of the task after training. Baliki and colleagues ${ }^{12}$ used fMRI to compare patients with chronic low back pain to normal controls subjected to thermal pain. They found a double disassociation with the patients with chronic back pain intensity correlating to the dorsal prefrontal cortex and the controls with thermal pain intensity correlating with the insula.

Baliki and colleagues ${ }^{5}$ compared the results of lidocaine patches in patients with chronic low back pain versus those with knee osteoarthritis. They used a finger span device that the patient used to continuously rate their pain while lying supine and undergoing fMRI. They demonstrated primarily activation of the mesial prefrontal lobes bilaterally in the patients with chronic low back pain. This activation decreased with lidocaine patches.

Giesecke and colleagues ${ }^{13}$ demonstrated augmented central pain processing in patients with chronic low back pain of unknown cause similar to patients with fibromyalgia.

DeCharms and colleagues ${ }^{7}$ used real-time MRI to give feedback about stimulation of the anterior cingulated gyrus to patients with chronic low back pain.

In our paradigm we were able to study subjects with both acute and chronic pain. We were able to demonstrate that a maneuver commonly used in clinical practice to augment pain (SLR maneuver) could be used during fMRI to study patients with low back and leg pain. We did not require any additional sophisticated equipment and the SLR maneuver could be easily performed by an assistant. Since low back pain represents one of the most common pain syndromes and the development of chronic low back pain is an increasing problem this paradigm should be helpful in studying these patients. Since patients with low back pain secondary to workman's compensation injury commonly go on to develop chronic pain they may be an ideal group of patients to study 
over time to determine what changes occur in the brain during the development of chronic pain. ${ }^{14,15}$

In summary, we were able to develop a paradigm to study patients with a common clinical pain syndrome using the SLR maneuver to vary the patients' pain during fMRI measurements. We plan to use this paradigm to study workman's compensation patients with lumbar and leg pain from herniated lumbar discs with longitudinal fMRI measurements, MR spectroscopy, and MR morphometric analyses. We hope this will provide insight into how the brain processes pain over time during the transition from acute to chronic pain.

\section{Disclosure}

The authors report no conflicts of interest in this work.

\section{References}

1. Atlas SJ, Chang Y, Kammann E, Keller RB, Deyo RA, Singer D. Long-term disability and return to work among patients who have a herniated lumbar disc: the effect of disability compensation. $J$ Bone Joint Surg Am. 2000;82:4-15.

2. Peyron R, Laurent B, García-Larrea L. Functional imaging of brain responses to pain: A review and meta-analysis. Neurophysiol Clin. 2000; $30: 263-288$

3. Tracey I. Imaging pain. Br J Anaesth. 2008;101:32-39.

4. Fairbank JC, Pynsent PB. The oswestry disability index. Spine. 2000;25: 2940-2952.

5. Baliki MN, Geha PY, Jabakhanji R, Harden N, Schnitzer TJ, Apkarian AV. A preliminary fMRI study of analgesic treatment in chronic back pain and knee osteoarthritis. Mol Pain. 2008;4:47.
6. Berman SM, Naliboff BD, Suyenobu B, et al. Reduced brainstem inhibition during anticipated pelvic visceral pain correlates with enhanced brain response to the visceral stimulus in women with irritable bowel syndrome. J Neurosci. 2008;28:349-359.

7. DeCharms RC, Maeda F, Glover GH, et al. Control over brain activation and pain learned by using real-time functional MRI. Proc Natl Acad Sci U S A. 2005;102:18626-18631.

8. Goebel R, Esposito F, Formisano E. Analysis of functional image analysis contest (FIAC) data with brainvoyager QX: From singlesubject to cortically aligned group general linear model analysis and self-organizing group independent component analysis. Hum Brain Mapp. 2006;27:392-401.

9. Talairach P, Tournoux J. A Stereotactic Coplanar Atlas of the Human Brain. Stuttgart, Germany: Thieme; 1988.

10. Deyo RA, Mirza SK, Martin BI. Back pain prevalence and visit rates: estimates from US national surveys. Spine. 2006;31:2724-2727.

11. Moseley GL. Widespread brain activity during an abdominal task markedly reduced after pain physiology education: fMRI evaluation of a single patient with chronic low back pain. Aust J Physiother. 2005;51: 49-52.

12. Baliki MN, Chialvo DR, Geha PY, et al. Chronic pain and the emotional brain: specific brain activity associated with spontaneous fluctuations of intensity of chronic back pain. J Neurosci. 2006;26:12165-12173.

13. Giesecke T, Gracely RH, Masilo AB, et al. Evidence of augmented central pain processing in idiopathic chronic low back pain. Arthritis Rheum. 2004;50:613-623.

14. Grachev ID, Fredrickson BE, Apkarian AV. Abnormal brain chemistry in chronic back pain: an in vivo proton magnetic resonance spectroscopy study. Pain. 2000;89:7-18.

15. Maghout-Juratli S, Franklin GM, Mirza SK, Wickizer TM, FultonKehoe D. Lumbar fusion outcomes in Washington state workers' compensation. Spine. 2006;31:2715-2723.
Journal of Pain Research

\section{Publish your work in this journal}

The Journal of Pain Research is an international, peer-reviewed, open access, online journal that welcomes laboratory and clinical findings in the fields of pain research and the prevention and management of pain. Original research, reviews, symposium reports, hypothesis formation and commentaries are all considered for publication.

\section{Dovepress}

The manuscript management system is completely online and includes a very quick and fair peer-review system, which is all easy to use. Visit http://www.dovepress.com/testimonials.php to read real quotes from published authors. 